20

\title{
Экспериментальные исследования модели зрительной системы человека
}

\author{
(C) Ю.С. Гулина, В.Я. Колючкин \\ МГтУ им. Н.Э. Баумана, \\ 105005 Москва, Россия \\ e-mail: julia-sg@yandex.ru, vkoluch@bmstu.ru \\ Поступила в редакцию 28.05.2019 г. \\ В окончательной редакции 28.05.2019 г. \\ Принята к публикации 18.06.2019 г.
}

\begin{abstract}
Изложены основные положения многоканальной модели зрительной системы человека, выполняющего функции оператора при наблюдении изображений, формируемых оптико-электронными приборами. Предложена методика экспериментальных исследований модели зрительной системы человека. Приведены результаты экспериментальных исследований, выполненных с целью уточнения параметров модели зрительной системы. Удовлетворительное совпадение расчетных и экспериментальных результатов свидетельствует об адекватности уточненной модели зрительной системы человека.
\end{abstract}

Ключевые слова: пространственная передаточная функция, временная передаточная функция, пространственный фильтр, помеха.

DOI: $10.21883 /$ OS.2019.10.48367.166-19

\section{Введение}

Особенностью оптико-электронных приборов (ОЭП), предназначенных для наблюдения объектов, в том числе телевизионных и тепловизионных приборов, а также приборов ночного видения, является то, что получателем информации, содержащейся в изображениях, формируемых этими приборами, является человек [1,2]. При наблюдении изображений, формируемых ОЭП наблюдения, человек выполняет функцию оператора, принимающего решение о наличии в угловом поле прибора изображений интересующих его объектов, а также отнесении обнаруженных объектов к определенному классу. Качество ОЭП наблюдения оценивается вероятностными показателями обнаружения и распознавания человеком-оператором изображений, наблюдаемых объектов. Поэтому математическая модель зрительной системы человека, с позиций разработчика ОЭП наблюдения, должна обеспечивать расчет вероятностных показателей обнаружения и распознавания изображений объектов, формируемых ОЭП. Анализу свойств зрительного восприятия и разработке математической модели зрительной системы человека в такой постановке задачи посвящен ряд публикаций [3-10].

В работе [11] на основе обобщения и анализа известных подходов к описанию зрительной системы предложена математическая модель, в которой отражены особенности преобразования оптических сигналов на этапе предварительной, первичной и вторичной обработки зрительных образов с учетом как внешних, так и внутренних помех. Результаты экспериментов в целом подтвердили адекватность предложенной математической модели. Однако дальнейшие исследования показали необходимость уточнения параметров модели, а также некоторых математических соотношений, выведенных при упрощающих допущениях.

Цель исследований - уточнение параметров предложенной авторами математической модели зрительной системы человека-оператора.

\section{Описание математической модели зрительной системы человека-оператора}

Рассмотрим основные положения предложенной математической модели зрительной системы. Предполагается, что человек-оператор наблюдает статическое изображение, формируемое ОЭП наблюдения на экране дисплея с частотой кадров $v_{k}$. Изображение содержит аддитивную помеху, представляющую собой однородное случайное поле, подчиняющееся нормальному закону. На этапе первичной обработки зрительных образов, осуществляемой в глазах, в математической модели учитываются:

- линейные искажения, которые вносит оптическая система (ОС) глаза;

- эффекты, возникающие при взаимодействии нейронов сетчатки, в том числе вычитание среднего уровня сигнала, регистрируемого рецепторами, и фильтрацию высоких пространственных частот (латеральное торможение);

- инерционность зрения;

- помехи типа фотонного и нейронного шума.

Отличительной особенностью этой модели является то, что на этапе первичной обработки изображений 
зрительная система представляется совокупностью параллельно включенных приемников, в каждом из которых производится пространственная фильтрация и последующее обнаружение составляющих сигнала, которые определяются значениями коэффициентов разложения изображения объектов в ортогональном базисе финитных гармонических функций. Теоретической предпосылкой такой многоканальной модели является возможность представления сигнала, описываемого пространственным распределением приращения яркости объекта относительно равномерного фона со значением яркости $\bar{L}$, выражением вида

$$
\begin{aligned}
& \Delta L\left(\varphi_{x}, \varphi_{y}\right)=\frac{\Delta L_{M}}{A_{\varphi 0}} \operatorname{rect}\left(\frac{\varphi_{x}}{l_{\varphi x}}, \frac{\varphi_{y}}{l_{\varphi y}}\right)\left\{\tilde{L}_{N}(0,0)\right. \\
& +3 \sum_{\substack{m=0 \\
n=-\infty \\
m \neq n=0}}^{\infty}\left|\tilde{L}_{N}\left(\frac{m}{l_{\varphi x}}, \frac{n}{l_{\varphi y}}\right)\right| \cos \left[2 \pi\left(\frac{\varphi_{x} m}{l_{\varphi x}}+\frac{\varphi_{y} n}{l_{\varphi y}}\right)\right. \\
& \left.\left.+\phi\left(\frac{m}{l_{\varphi x}}, \frac{n}{l_{\varphi y}}\right)\right]\right\},
\end{aligned}
$$

где $\varphi_{x}=x / L_{D}$ и $\varphi_{y}=y / L_{D}$ - выраженные в градусах угловые координаты точек поверхности объекта при дистанции $L_{D}$ наблюдения; $A_{\varphi 0}=l_{\varphi x} l_{\varphi y}-$ габаритная площадь объекта; $\Delta L_{M}-$ максимальное значение приращения яркости поверхности объекта;

$$
\left|\tilde{L}_{N}\left(\frac{m}{l_{\varphi x}}, \frac{n}{l_{\varphi y}}\right)\right| \quad \text { и } \quad \phi\left(\frac{m}{l_{\varphi x}}, \frac{n}{l_{\varphi y}}\right)
$$

- значение модуля и фазы фурье-спектра функции, описывающей нормированное распределение яркости объекта на угловых пространственных частотах, кратных значениям, обратным габаритным угловым размерам $l_{\varphi x}$ и $l_{\varphi y}$. Выражение (1) описывает разложение сигнала от объекта в ортогональном базисе финитных гармонических функций, в котором коэффициенты разложения можно интерпретировать как признаки, используемые для обнаружения и распознавания образов объектов. Селекция признаков осуществляется пространственными фильтрами, передаточные функции (ПФ) которых имеют вид

$$
\begin{aligned}
& \tilde{H}_{m n}\left(v_{\varphi x}, v_{\varphi y}\right)=\frac{1}{2}\left\{\operatorname{sinc}\left[\pi l_{\varphi x}\left(v_{\varphi x}-\frac{m}{l_{\varphi x}}\right)\right]\right. \\
& \times \operatorname{sinc}\left[\pi l_{\varphi y}\left(v_{\varphi y}-\frac{n}{l_{\varphi y}}\right)\right]+\operatorname{sinc}\left[\pi l_{\varphi x}\left(v_{\varphi x}+\frac{m}{l_{\varphi x}}\right)\right] \\
& \left.\times \operatorname{sinc}\left[\pi l_{\varphi y}\left(v_{\varphi y}+\frac{n}{l_{\varphi y}}\right)\right]\right\} \exp \left[-i \phi^{*}\left(\frac{m}{l_{\varphi x}}, \frac{n}{l_{\varphi y}}\right)\right] .
\end{aligned}
$$

В силу ортогональности базисных функций такие признаки являются независимыми. Поэтому для расчета вероятности обнаружения изображения объекта челове- ком можно использовать формулу [12]

$$
P_{D}=1-\prod_{m, n}\left(1-P_{D}^{m n}\right)
$$

где $P_{m n}^{D}-$ вероятности обнаружения $m n-\mathrm{x}$ гармонических составляющих.

При принятом допущении о нормальном законе распределении помех вероятности $P_{m n}^{D}$ рассчитываются по формуле

$$
P_{D}^{m n}=\frac{1}{\sqrt{2 \pi}} \int_{\xi_{t}^{m n}}^{\infty} \exp \left[-\frac{\left(\xi-\mu_{m n}\right)^{2}}{2}\right] d \xi
$$

где порог обнаружения $\xi_{t}^{m n}$ определяется в соответствии с критерием максимума апостериорной вероятности при экспериментально определенном значении порогового отношения правдоподобия $\Lambda_{t}=120$, а именно

$$
\xi_{t}^{m n}=\frac{\ln \Lambda_{t}}{\mu_{m n}}+\frac{\mu_{m n}}{2} .
$$

Входящая в выражения (3) и (4) величина $\mu_{m n}$ представляет собой отношение сигнала к шуму (ОСШ) на выходе пространственного фильтра $m n$-го приемника, которое определяется как

$$
\mu_{m n}=S_{m n} / \sigma_{m n},
$$

где $S_{m n}-$ сигнальная составляющая, $\sigma_{m n}-$ среднеквадратическое отклонение (CКО) помех.

При известном значении контраста $C_{M}=\Delta L_{M} / \bar{L}$ изображения объекта, наблюдаемого на экране дисплея на равномерном фоне яркостью $\bar{L}$, и с учетом линейных искажений первичной обработки зрительной системы сигнальная составляющая на выходе $m n$-го пространственного фильтра описывается выражением

$$
S_{m n}=C_{M} \bar{n}_{p h}\left|\tilde{L}_{N S}\left(\frac{m}{l_{\varphi x}}, \frac{n}{l_{\varphi y}}\right)\right|\left|\tilde{H}_{E}\left(\frac{m}{l_{\varphi x}}, \frac{n}{l_{\varphi y}}\right)\right|,
$$

где $\bar{n}_{p h}-$ среднее число фотонов, регистрируемых фоторецепторами сетчатки за время $\tau_{V}=0.1 \mathrm{~s}$, которое характеризует инерционность зрительной системы;

$$
\left|\tilde{L}_{N S}\left(\frac{m}{l_{\varphi x}}, \frac{n}{l_{\varphi y}}\right)\right|=\left|\tilde{L}_{N}\left(\frac{m}{l_{\varphi x}}, \frac{n}{l_{\varphi y}}\right)\right| / A_{\varphi o}
$$

- амплитуда mn-й гармоники спектра сигнала, нормированная на габаритную площадь $A_{\varphi о}$ изображения объекта;

$$
\tilde{H}_{E}\left(\frac{m}{l_{\varphi x}}, \frac{n}{l_{\varphi y}}\right)=\tilde{H}_{O S}\left(\frac{m}{l_{\varphi x}}, \frac{n}{l_{\varphi y}}\right) \tilde{H}_{l a t}\left(\frac{m}{l_{\varphi x}}, \frac{n}{l_{\varphi y}}\right)
$$

- значение ПФ глаза на пространственных частотах $m / l_{\varphi x}$ и $n / l_{\varphi y} ; \tilde{H}_{O S}\left(\frac{m}{l_{\varphi x}}, \frac{n}{l_{\varphi v}}\right)$ - значение ПФ ОС глаза; $\tilde{H}_{l a t}\left(\frac{m}{l_{\varphi x}}, \frac{n}{l_{\varphi y}}\right)$ - значение ПФ линейного звена, учитывающего эффект латерального торможения, возникающего между нейронами сетчатки глаза. 
Следует отметить, что среднее число фотонов, регистрируемых рецепторами глаза, выражается через среднее значение $\bar{L}$ освещенности на сетчатке в соответствии с формулой

$$
\bar{n}_{p h}=\frac{\bar{E} \eta q_{r}}{k_{E}},
$$

где $\eta$ квантовая эффективность фоторецепторов; $q_{r} \mathrm{~cm}^{2}$ - площадь рецептивного поля ганглиозных клеток сетчатки; $k_{E}=10^{-11} \mathrm{~lx} \cdot \mathrm{cm} /$ photon - коэффициент пересчета значений освещенности, выраженной в люкcax, в число фотонов [13].

Так как результирующая помеха включает внешнюю аддитивную помеху, присутствующую в изображении на экране дисплея ОЭП наблюдения, а также помехи в виде фотонного и нейронного шума, которые не коррелированы между собой, то СКО помехи на выходе пространственного фильтра $m n$-го приемника определяется выражением

$$
\sigma_{m n}=\sqrt{\sigma_{e m n}^{2}+\sigma_{p h m n}^{2}+\sigma_{n m n}^{2}}
$$

где $\sigma_{e m n}^{2}, \sigma_{p h m n}^{2}$ и $\sigma_{n m n}^{2}-$ значения дисперсии внешнего, фотонного и нейронного шума соответственно.

Были выведены математические выражения для расчета дисперсий каждой из составляющих помехи. В частности, выражение для дисперсии внешней аддитивной помехи имеет вид

$$
\begin{aligned}
\sigma_{e m n}^{2}= & \frac{\bar{n}_{p h}^{2} \sigma_{e N}^{2} A_{\varphi p}}{v_{k}} \iiint_{-\infty}^{\infty}\left|\tilde{H}_{V}\left(v_{\varphi x}, v_{\varphi y}, v_{t}\right)\right|^{2} \\
& \times\left|\tilde{H}_{m n}\left(v_{\varphi x}, v_{\varphi y}\right)\right|^{2} d v_{\varphi x} d v_{\varphi y} d v_{t},
\end{aligned}
$$

где $\sigma_{e N}=\sigma_{L} / \bar{L}$ - нормированное значение СКО внешней аддитивной помехи; $A_{\varphi p}=a_{\varphi} b_{\varphi}-$ площадь корреляции аддитивной помехи, равная площади в угловой мере одного пикселя на экране дисплея; $\tilde{H}_{V}\left(v_{\varphi x}, v_{\varphi y}, v\right)-$ пространственно-временна́я ПФ зрительной системы, зависящая от угловых пространственных частот $v_{\varphi x}$ и $v_{\varphi y}$, а также временной частоты $v_{t} ; \tilde{H}_{m n}\left(v_{\varphi x}, v_{\varphi y}\right)-\Pi \Phi$ пространственного фильтра $m n$-го приемника.

Пространственно-временная ПФ зрительной системы в свою очередь определяется как

$$
\begin{aligned}
\tilde{H}_{V}\left(v_{\varphi x}, v_{\varphi y}, v_{t}\right)= & \tilde{H}_{O S}\left(v_{\varphi x}, v_{\varphi y}\right) \tilde{H}_{V}\left(v_{\varphi x}, v_{\varphi y}, v_{t}\right) \\
& \times \tilde{H}_{T}\left(v_{t}\right) \tilde{H}_{L a t}\left(v_{\varphi x}, v_{\varphi y}, v_{t}\right),
\end{aligned}
$$

где $\tilde{H}_{O S}\left(v_{\varphi x}, v_{\varphi y}\right)$ - ПФ ОС глаза; $\tilde{H}_{T}\left(v_{t}\right)-$ временная ПФ зрительной системы; $\tilde{H}_{L a t}\left(v_{\varphi x}, v_{\varphi y}, v_{t}\right)-$ пространственно-временная ПФ латерального торможения.

ПФ ОС глаза описывается выражением

$$
\tilde{H}_{O S}\left(v_{\varphi x}, v_{\varphi y}\right)=\tilde{H}_{O S}\left(v_{\varphi r}\right)=\exp \left(-2 \pi^{2} \sigma^{2} v_{\varphi r}^{2}\right),
$$

где $v_{\varphi r}=\sqrt{v_{\varphi x}^{2}+v_{\varphi y}^{2}}, \sigma$ - параметр аппроксимации, который выражается в угловых градусах и рассчитывается по эмпирической формуле

$$
\sigma=\sqrt{\sigma_{0}^{2}+\left(C_{a b} d\right)^{2}} / 60,
$$

где $\sigma_{0}=0.5 \operatorname{arc~min}, C_{a b}=0.8 \operatorname{arc~min} / \mathrm{cm} ; d, \mathrm{~cm}$ - диаметр зрачка глаза, также определяемый по эмпирической формуле

$$
d=[5-\operatorname{th}(\lg \bar{L})] / 10 .
$$

Временная ПФ, характеризующая инерционность зрительной системы, описывается выражением

$$
\tilde{H}_{T}\left(v_{t}\right)=\frac{1}{\sqrt{1+\left(2 \pi v_{t} \tau_{V}\right)^{2}}} \exp \left[-i \operatorname{arctg}\left(2 \pi v_{t} \tau_{V}\right)\right]
$$

В предложенной математической модели зрительной системы учтено, что эффект латерального торможения, возникающий при взаимодействии нейронов сетчатки, зависит от возможных временных флуктуаций изображения, регистрируемого фоторецепторами. Даже при статичном образе объекта реализации внешней помехи, присутствующей в изображении на экране дисплея ОЭП наблюдения, изменяются от кадра к кадру. Чтобы учесть этот фактор в модели зрительной системы, выражение пространственно-временной ПФ латерального торможения, входящее в формулу (11), представлено в виде

$$
\tilde{H}_{L a t}\left(v_{\varphi r}, v_{t}\right)=1-\tilde{H}_{T 1}\left(v_{t}\right)\left[1-\tilde{H}_{l a t}\left(v_{\varphi r}\right)\right],
$$

где $\tilde{H}_{T 1}(v)$ - временная ПФ, характеризующая зависимость эффекта латерального торможения от временных характеристик регистрируемого изображения; $\tilde{H}_{\text {lat }}\left(v_{\varphi r}\right)$ - пространственная ПФ, описывающая эффект латерального торможения.

Входящие в выражение (15) ПФ определяются следующими формулами:

$$
\begin{gathered}
\tilde{H}_{T 1}\left(v_{t}\right)=\frac{1}{\left[1+\left(2 \pi \tau_{1} v_{t}\right)^{2}\right]^{2}} \exp \left[-i 4 \operatorname{arctg}\left(2 \pi \tau_{1} v_{t}\right)\right], \\
\tilde{H}_{\text {lat }}\left(v_{\varphi r}\right)=\frac{i 2 \pi v_{\varphi r} r_{l a t}}{1+i 2 \pi v_{\varphi r} r_{l a t}}
\end{gathered}
$$

В ранее опубликованной работе были приняты следующие значения параметров, входящих в формулы (16) и (17): $\tau_{1}=1.35 \cdot 10^{-2} \mathrm{~s} ; r_{\text {lat }}=0.1 \mathrm{deg}$.

Выражения, выведенные для расчета дисперсии помех типа фотонного и нейронного шума, соответственно имеют вид

$$
\begin{gathered}
\sigma_{p h m n}^{2}=\bar{n}_{p h}^{2} \frac{k_{E}\left(180^{\circ}\right)^{2} 2 \tau_{V}}{\pi^{3} \eta \bar{L} d^{2} / 4} \iiint_{-\infty}^{\infty}\left|\tilde{H}_{l a t}\left(v_{\varphi x}, v_{\varphi y}\right)\right|^{2} \\
\times\left|\tilde{H}_{T}\left(v_{t}\right)\right|^{2}\left|\tilde{H}_{m n}\left(v_{\varphi x}, v_{\varphi y}\right)\right|^{2} d v_{\varphi x} d v_{\varphi y} d v_{t}, \\
\sigma_{n m n}^{2}=\bar{n}_{p h}^{2} \tilde{R}_{n M} \iiint_{-\infty}^{\infty}\left|\tilde{H}_{T}\left(v_{t}\right)\right|^{2} \\
\times\left|\tilde{H}_{m n}\left(v_{\varphi x}, v_{\varphi y}\right)\right|^{2} d v_{\varphi x} d v_{\varphi y} d v_{t},
\end{gathered}
$$


где $\tilde{R}_{n N}-$ спектральная плотность нейронного шума, значение которой по результатам других авторов ранее было принято равным $\tilde{R}_{n N}=6.0 \cdot 10^{-4} \mathrm{deg}^{2} \cdot \mathrm{sec}$.

Выражение (19) после интегрирования с учетом формул (2) и (14) можно представить в виде

$$
\sigma_{n m n}^{2}=\frac{\bar{n}_{p h}^{2} \tilde{R}_{n n N}}{4 A_{\varphi 0} \tau_{V}} .
$$

При допущении о том, что функции $\operatorname{sinc}^{2}\left[\pi l_{\varphi x}\left(v_{\varphi x}-m / l_{\varphi x}\right)\right] \quad$ и $\operatorname{sinc}^{2}\left[\pi l_{\varphi y}\left(v_{\varphi y}-m / l_{\varphi x}\right)\right]$, входящие в формулы (10), (19) и (20), имеют вид относительно узких пиков, были получены следующие приближенные выражения для расчета дисперсий внешней и фотонной помехи:

$$
\begin{gathered}
\sigma_{e m n}^{2} \approx \frac{\bar{n}_{p h}^{2} \sigma_{e N}^{2} A_{\varphi p}}{2 A_{\varphi 0} 2 \tau_{V} v_{k}}\left|\tilde{H}_{O S}\left(\frac{m}{l_{\varphi x}}, \frac{n}{l_{\varphi y}}\right)\right|^{2}\left|\tilde{H}_{1 \text { lat }}\left(\frac{m}{l_{\varphi x}}, \frac{n}{l_{\varphi y}}\right)\right|^{2}, \\
\sigma_{p h m n}^{2} \approx \bar{n}_{p h}^{2} \frac{k_{E}\left(180^{\circ}\right)^{2}}{\pi^{3} \eta \bar{L} d^{2} / 4}\left|\tilde{H}_{1 \text { lat }}\left(\frac{m}{l_{\varphi x}}, \frac{n}{l_{\varphi y}}\right)\right|^{2},
\end{gathered}
$$

где

$$
\begin{gathered}
\left|\tilde{H}_{1 l a t}\left(\frac{m}{l_{\varphi x}}, \frac{n}{l_{\varphi y}}\right)\right|^{2}=1-1.206\left[1-\tilde{H}_{\text {lat }}\left(\frac{m}{l_{\varphi x}}, \frac{n}{l_{\varphi y}}\right)\right] \\
+0.764\left[1-\tilde{H}_{l a t}\left(\frac{m}{l_{\varphi x}}, \frac{n}{l_{\varphi y}}\right)\right]^{2} .
\end{gathered}
$$

С учетом формул (7), (21)-(23) приближенное выражение для расчета ОСШ для $m n$-й гармоники сигнала представляется выражением

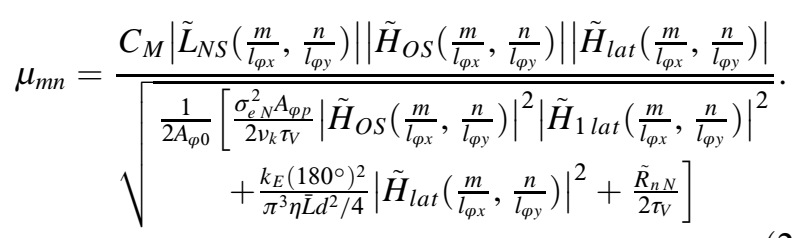

Выведенные выражения позволяют рассчитать вероятность обнаружения человеком-оператором изображения объекта, формируемого на экране дисплея ОЭП наблюдения. В частности, описанная выше математическая модель может быть использована и для оценки вероятности распознавания изображений объектов по методике Джонсона.

Для изучения свойств зрительного восприятия физиологи используют функцию контрастной чувствительности (ФКЧ), определяющей зависимость от пространственной частоты $v_{\varphi x}=m / l_{\varphi x}$ величины $S\left(v_{\varphi x}\right)=$ $=1 / C^{*}\left(v_{\varphi x}\right)$, которая является обратной значению контраста $C^{*}$, при котором с вероятностью 0.5 обнаруживается финитный гармонический объект. Для принятого модельного описания вероятность обнаружения, равная 0.5 , достигается при значении ОСШ $\mu^{*}=3.1$.

В результате замены в выражении (25) $C_{M}$ на $C^{*}, \mu_{m n}$ на $\mu^{*}$ и учета того, что для финитного гармонического объекта $\tilde{L}_{O S}\left(\frac{m}{l_{\varphi x}}, \frac{n}{l_{\varphi v}}\right)=\frac{1}{2}$, получена следующая формула для расчета ФКЧ:

$$
\begin{aligned}
& S\left(\frac{m}{l_{\varphi x}}, 0\right)=
\end{aligned}
$$

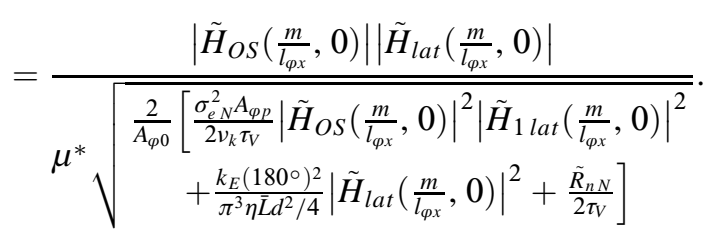

Проведенные экспериментальные исследования по измерению ФКЧ зрительной системы в целом подтвердили адекватность предложенной многоканальной математической модели зрительной системы человека-оператора. Однако дальнейшие исследования выявили необходимость уточнения некоторых параметров модели. Конкретно, была поставлена задача уточнения параметров $\tau_{1}$ и $r_{l a t}$, характеризующих эффект латерального торможения, значения нормированной спектральной плотности $\tilde{R}_{n n N}$ нейронного шума и коэффициента $k_{E}$, используемого для пересчета значений освещенности, выраженной в люксах, в число фотонов. Кроме этого, поставлена задача определения границ применимости приближенных формул (22) и (23), выведенных для расчета дисперсии внешней помехи и фотонного шума. Поставленная задача решалась путем сравнения результатов экспериментальных исследований и расчетов по формулам, описывающим математическую модель зрительной системы.

\section{Методика экспериментальных исследований}

Экспериментальные исследования проводились путем оценки вероятности обнаружения человеком чернобелых изображений тест-объектов в виде периодических вертикально ориентированных решеток с синусоидальным распределением яркости, у которых изменялись размеры, пространственная частота, контраст относительно равномерного фона и уровень внешней аддитивной помехи. В экспериментах принимали участие 3 обученных оператора, а вероятность обнаружения оценивалась частотой принятия правильных решений при 5 предъявлениях каждого изображения тест-объекта. На основании сравнения расчетных и экспериментальных результатов производилось уточнение параметров математической модели.

Изображения тест-объектов формировались на экране монитора компьютера с помощью специально разработанного программного обеспечения. Монитор позволял отображать образы объектов, которые имели 255 градаций яркости при паспортном среднем значении яркости монитора экрана, равном $120 \mathrm{~cd} / \mathrm{m}^{2}$. Обеспечивалось формирование на экране монитора зашумленных изображений тест-объектов в пределах окна на экране 
дисплея размером $170 \times 150$ пикселей с частотой кадров $25 \mathrm{~Hz}$. При наблюдении изображений операторы располагались таким образом, чтобы расстояние от глаз до экрана, примерно равнялось $600 \mathrm{~mm}$.

На этапе подготовки к проведению экспериментов с помощью люксметра была измерена зависимость яркости экрана от числа градаций сигнала, поступающего от компьютера, на котором моделировалось изображение тест-объектов. Эта зависимость была аппроксимирована уравнением прямой линии, имеющей вид

$$
L=70+0.98(K-100),
$$

где $K$ - число градаций среднего уровня цифрового сигнала, которое в экспериментах изменялось от 100 до 255. Относительная погрешность такой аппроксимации не превышала $15 \%$.

Кроме этого, при сравнении результатов экспериментов с расчетными результатами были учтены линейные искажения, которые вносит экран монитора. Такие искажения приводят, во-первых, к снижению контраста изображения синусоидальных тест-объектов, и, во-вторых, к „окраске“ внешней аддитивной помехи, для которой была принята модель квазибелого шума. Чтобы учесть эти факторы, формулы (6), (9) и (18), которые использовались при вычислении ОСШ, были представлены в виде

$$
\begin{aligned}
& S_{m n}=C_{M} \bar{n}_{p h}\left|\tilde{L}_{N S}\left(\frac{m}{l_{\varphi x}}, \frac{n}{l_{\varphi y}}\right)\right|\left|\tilde{H}_{D}\left(\frac{m}{l_{\varphi x}}, \frac{n}{l_{\varphi y}}\right)\right| \\
& \quad \times\left|\tilde{H}_{E}\left(\frac{m}{l_{\varphi x}}, \frac{n}{l_{\varphi y}}\right)\right|, \\
& \sigma_{e m n}^{2}=\frac{\bar{n}_{p h}^{2} \sigma_{e N}^{2} A_{\varphi p}}{2 A_{\varphi 0} 2 \tau_{V} v_{k}} \iint_{-\infty}^{\infty}\left|\tilde{H}_{D}\left(v_{\varphi x}, v_{\varphi y}\right)\right|^{2} \\
& \times\left|\tilde{H}_{1 \text { lat }}\left(v_{\varphi x}, v_{\varphi y}\right)\right|^{2}\left|\tilde{H}_{m n}\left(v_{\varphi x}, v_{\varphi y}\right)\right|^{2} d v_{\varphi x} d v_{\varphi y}, \\
& \sigma_{p h m n}^{2}=\bar{n}_{p h}^{2} \frac{k_{E}\left(180^{\circ}\right)^{2}}{\pi^{3} \eta \bar{L} d^{2} / 4} \int_{-\infty}^{\infty}\left|\tilde{H}_{D}\left(v_{\varphi x}, v_{\varphi y}\right)\right|^{2} \\
& \times\left|\tilde{H}_{1 \text { lat }}\left(v_{\varphi x}, v_{\varphi y}\right)\right|^{2}\left|\tilde{H}_{m n}\left(v_{\varphi x}, v_{\varphi y}\right)\right|^{2} d v_{\varphi x} d v_{\varphi y},
\end{aligned}
$$

где $\left|\tilde{H}_{D}\left(v_{\varphi x}, v_{\varphi y}\right)\right|$ - пространственная ПФ экрана монитора.

Принято, что пиксели экрана дисплея имеют квадратную форму с угловым размером $A_{\varphi}=b_{\varphi}=0.024 \mathrm{deg}$. При этом ПФ экрана монитора описывается выражением

$$
\tilde{H}_{D}\left(v_{\varphi x}, v_{\varphi y}\right)=\operatorname{sinc}\left(\pi v_{\varphi x} \alpha_{\varphi}\right) \operatorname{sinc}\left(\pi v_{\varphi y} \alpha_{\varphi}\right) .
$$

\section{Экспериментальная оценка значения нормированной спектральной плотности нейронного шума}

При проведении экспериментов перед операторами ставилась задача обнаружения тест-объекта при измене-

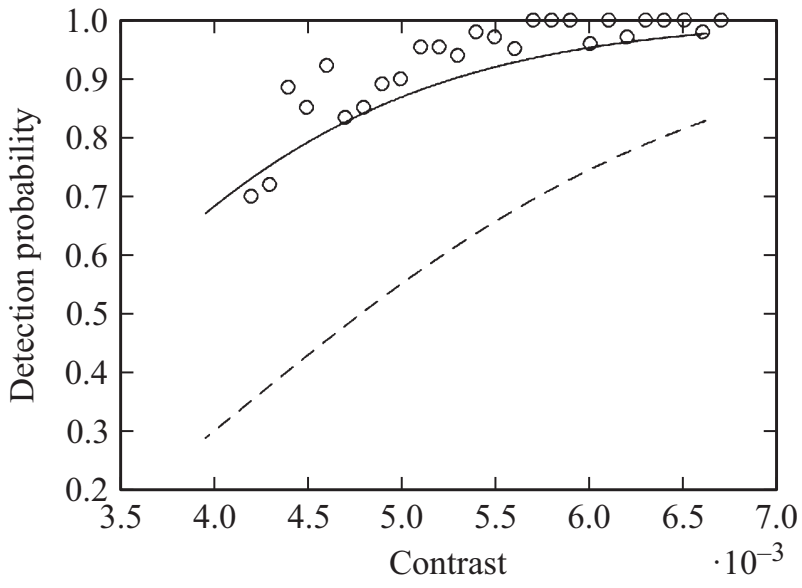

Рис. 1. Зависимость вероятности обнаружения гармонической решетки от контраста: о - результаты экспериментов, --- график расчетной зависимости при $\tilde{R}_{n N}=6 \cdot 10^{-8} \mathrm{deg}^{2} \cdot \mathrm{s}$, - график расчетной зависимости при $\tilde{R}_{n N}=6 \cdot 10^{-9} \mathrm{deg}^{2} \cdot \mathrm{s}$.

нии значения контраста в изображении. Вероятность обнаружения оценивалась частотой принятия правильных решений при пяти предъявлениях каждого изображения. При частоте правильных ответов, больших 0.5, оператору предъявлялось изображение с менышим контрастом, который изменялся путем увеличения уровня яркости фона на одну градацию.

Для оценки значения спектральной плотности нейронного шума требовалось создать условия, при которых эта помеха была бы больше или соизмерима с фотонным шумом и аддитивной помехой. Так как дисперсия фотонного шума обратно пропорциональна значению яркости фона, то для минимизации данной помехи экспериментальные исследования проводились при максимально возможных уровнях яркости фона. Но в условиях реализуемого на экране дисплея диапазона значений контраста, который ограничен 255 градациями яркости, при исключении внешней аддитивной помехи изображение тест-объектов обнаруживалось с вероятностью, близкой к единице. Поэтому эксперименты проводились при добавлении в изображение тест-объекта внешней аддитивной помехи, имеющей вид „квазибелого шума“ с нулевым средним значением и относительным СКО, равным $\sigma_{e N}=0.05$.

Чтобы уменьшить влияние неопределенности значений параметров, характеризующих эффект латерального торможения, в этой серии экспериментов использовался тест-объект в виде синусоидальной решетки с пространственной частотой $v_{\varphi x}=6 \mathrm{deg}^{-1}$. При этом в пределах угловых размеров тест-объекта, равных $1.00 \times 0.57 \mathrm{deg}$, укладывалось 6 периодов.

На рис. 1 представлены результаты экспериментов и расчетов зависимости вероятности обнаружения от контраста тест-объекта.

Из рис. 1 следует, что при ранее принятом значении нормированной спектральной плотности нейронно- 
го шума, равном $\tilde{R}_{n N}=6.0 \cdot 10^{-8} \mathrm{deg}^{2} \cdot \mathrm{s}$, график расчетной зависимости сильно занижен по сравнению с экспериментальными результатами. Но при значении $\tilde{R}_{n N}=6.0 \cdot 10^{-9} \mathrm{deg}^{2} \cdot \mathrm{s}$ отличие расчетной зависимости от результатов экспериментов не превышает $10 \%$. Поэтому в дальнейшем в модели зрительной системы человека принято именно это значение нормированной спектральной плотности нейронного шума.

\section{Экспериментальная оценка значений параметров латерального торможения}

Выше отмечено, что в выражения (15)-(17) для пространственно-временной ПФ, описывающей эффект латерального торможения зрительной системы человека, входят параметры, значения которых приняты равными $r_{\text {lat }}=0.1 \mathrm{deg}$ и $\tau_{1}=1.35 \cdot 10^{-2} \mathrm{~s}$.

Следует отметить, что при $r_{l a t}=0.1 \mathrm{deg}$ ФКЧ имеет экстремум в области значения пространственной частоты $v_{\varphi x}=4 \mathrm{deg}^{-1}$. Но на основе экспериментальных измерений ФКЧ, изложенных ниже в настоящей работе, а также публикаций других авторов, установлено, что максимум ФКЧ лежит в окрестности пространственной частоты, равной $v_{\varphi x}=5 \mathrm{deg}^{-1}$. При таком условии значение параметра, характеризующего крутизну ПФ $\tilde{H}_{\text {lat }}\left(v_{\varphi r}\right)$, выбрано равным $r_{\text {lat }}=0.05 \mathrm{deg}$.

Параметр $\tau_{1}$ определяет степень влияния эффекта латерального торможения при наличии временных флуктуаций в наблюдаемом человеком изображении. Как отмечено выше, при наблюдении на экране дисплея статичных изображений объектов, содержащих изменяющиеся от кадра к кадру реализации аддитивной помехи, временные свойства латерального торможения влияют на уровень зрительного восприятия этой помехи.

Для уточнения значения параметра $\tau_{1}$ были проведены эксперименты, которые заключались в обнаружении зашумленных изображений гармонических тест-объектов. Так как временные свойства латерального торможения проявляются в области низких пространственных частот, то в экспериментах использовался тест-объект с частотой, равной ей в настоящей работе. Также из публикаций других авторов установлено, что максимум ФКЧ лежит в окрестности пространственной частоты, равной $v_{\varphi x}=5 \mathrm{deg}^{-1}$. При этом в пределах угловых размеров тест-объекта, равных $1.00 \times 0.57 \mathrm{deg}$, укладывался только один период решетки.

Перед операторами, участвовавшими в экспериментах, ставилась задача обнаружения тест-объекта при увеличении значения дисперсии аддитивного шума при постоянном контрасте $C$ синусоидальной решетки, равном двум градациям яркости. Вероятность обнаружения оценивалась частотой принятия правильных решений при 5 предьявлениях каждого изображения. При частоте правильных ответов, больших 0.5 , оператору предьявлялось изображение с большим на одну градацию СКО аддитивной помехи.

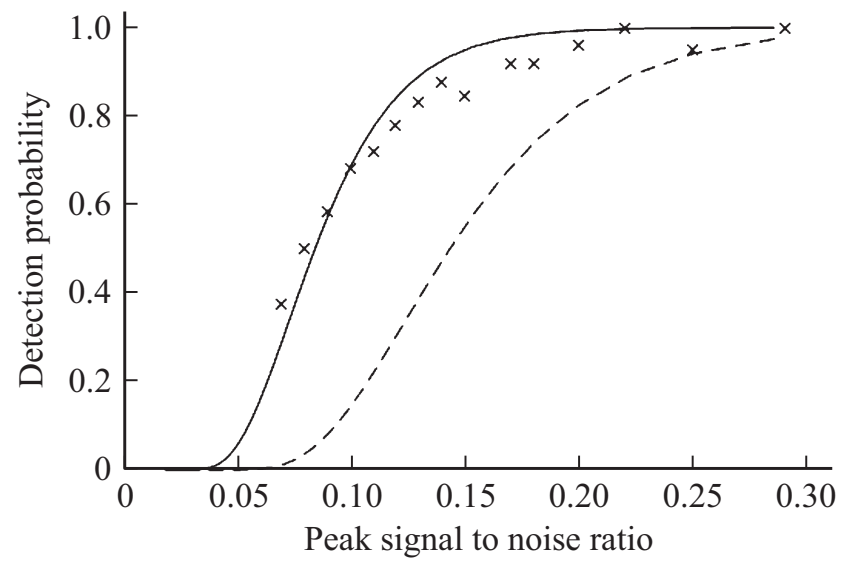

Рис. 2. Зависимость вероятности обнаружения от пикового ОСШ: $\times$ - результаты экспериментов, --- - график расчетной зависимости при $\tau_{1}=1.35 \cdot 10^{-2} \mathrm{~s},--$ график расчетной зависимости при $\tau_{1}=2.0 \cdot 10^{-3} \mathrm{~s}$.

На рис. 2 представлены результаты экспериментов и расчетов в виде зависимости вероятности обнаружения от пикового ОСШ, которое определялось как $\mu_{p}=C / \sigma_{e N}$. Результаты расчетов получены для значений $\tau_{1}=1.35 \cdot 10^{-2} \mathrm{~s}$ и $\tau_{1}=2.0 \cdot 10^{-3} \mathrm{~s}$. Как следует из рис. 2 , график, рассчитанный при $\tau_{1}=2.0 \cdot 10^{-3} \mathrm{~s}$, в значительно большей степени согласуется с результатами экспериментов. Поэтому в дальнейшем в математической модели предлагается использовать это значение параметра латерального торможения.

При таком значении параметра $\tau_{1}$ выражение (23), описывающее квадрат модуля усредненной по времени ПФ латерального торможения и входящее в выражение (28) для расчета дисперсии внешней помехи, имеет вид

$$
\begin{aligned}
\left|\tilde{H}_{1 \text { lat }}\left(v_{\varphi x}, v_{\varphi y}\right)\right|^{2}=1 & -1.855\left[1-\tilde{H}_{l a t}\left(v_{\varphi x}, v_{\varphi y}\right)\right] \\
& +0.95\left[1-\tilde{H}_{l a t}\left(v_{\varphi x}, v_{\varphi y}\right)\right]^{2} .
\end{aligned}
$$

Чтобы проиллюстрировать особенности фильтрации внешней помехи, реализации которой изменяются во времени с частотой кадров, на рис. 3 приведены графики функций $\left|\tilde{H}_{1 \text { lat }}\left(v_{\varphi x}, v_{\varphi y}\right)\right|^{2}$ и $\left|\tilde{H}_{l a t}\left(v_{\varphi x}, v_{\varphi y}\right)\right|^{2}$.

Из графиков этих функций, а также из формул (27)-(29) следует, что внешняя аддитивная помеха в отличие от статического сигнала от объекта и фотонного шума в меньшей степени подавляется в области низких пространственных частот.

\section{Экспериментальная оценка СКО фотонного шума и границ применимости приближенных формул модели}

Оценка СКО фотонного шума производилась путем сравнения результатов измерения ФКЧ с расчетны- 


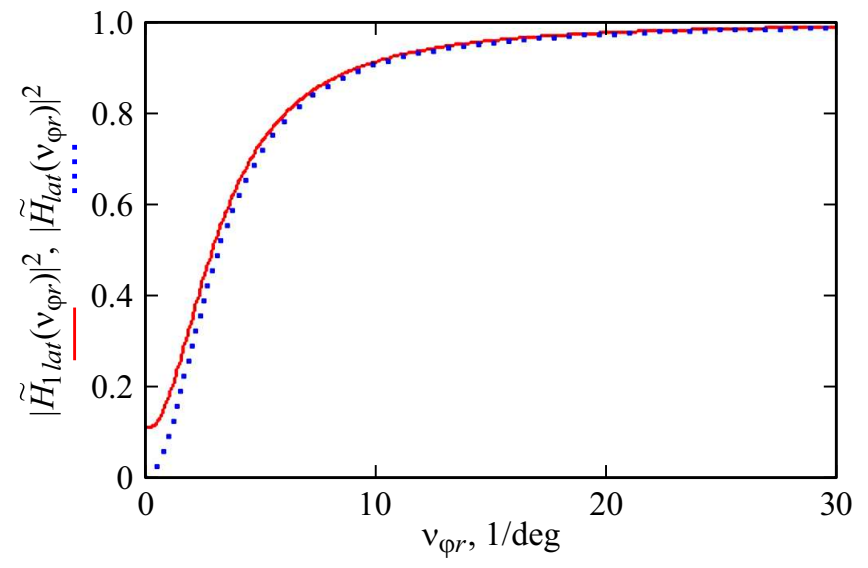

Рис. 3. Графики функций $\left|\tilde{H}_{1 \text { lat }}\left(v_{\varphi x}, v_{\varphi y}\right)\right|^{2}$ и $\left|\tilde{H}_{l a t}\left(v_{\varphi x}, v_{\varphi y}\right)\right|^{2}$.

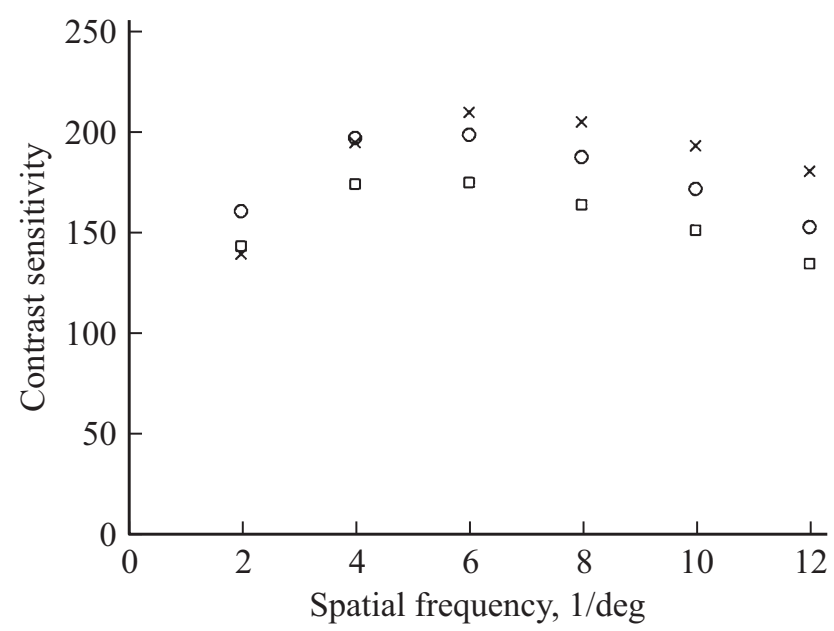

Рис. 4. Расчетные и экспериментальные значения ФКЧ: $\times$ - экспериментальные значения, $\square-$ расчетные значения при $k_{E}=10^{-11} \mathrm{~lx} \cdot \mathrm{cm} /$ photon, ○- расчетные значения при $k_{E}=6.1^{-12} 1 \mathrm{x} \cdot \mathrm{cm} /$ photon.

ми значениями. При этом в расчетах использовались уточненные выше значения параметров математической модели. Чтобы обеспечить условия, при которых преобладающей помехой является фотонный шум, требовалось минимизировать внешнюю аддитивную помеху. Из-за ограничений диапазона градаций яркости экрана монитора при проведении экспериментов полностью эту помеху исключить не удавалось. Это обусловлено тем, что при отсутствии внешней помехи изображение тест-объекта обнаруживалось с вероятностью, больше 0.5 , в то время как значения ФКЧ определяется при вероятности, равной 0.5. В связи с этим операторам на экране монитора компьютера предъявлялись зашумленные изображения тест-объектов, содержавшие гауссову помеху с нулевым средним значением и относительной дисперсией, равной $\sigma_{e N}=0.02$. В изображениях тестобъектов в виде гармонических решеток с размерами $0.50 \times 0.29 \mathrm{deg}$ изменялись пространственная частота и контраст. Изменение контраста осуществлялось варьированием яркости фона в диапазоне градаций яркости от 120 до 200, что соответствовало значениям яркости экрана от 88 до $160 \mathrm{~cd} / \mathrm{m}^{2}$.

На рис. 4 представлены результаты экспериментов и расчетов ФКЧ в диапазоне пространственных частот от 2 до $12 \mathrm{deg}^{-1}$. Расчетные значения, отмеченные квадратами, получены при расчете по формуле (26) с учетом линейных искажений экрана монитора и при значении коэффициента $k_{E}=10^{-11} \mathrm{~lx} \cdot \mathrm{cm} /$ photon, который был ранее принят в математической модели зрительной системы на основе данных А. Роуз. Расчетные значения в области пространственных частот от 4 до $12 \mathrm{deg}^{-1}$ оказались меньше экспериментальных, причем отличие достигало $28 \%$, что свидетельствует о завышенной оценке СКО фотонного шума.

По данным, приведенным в [14], значение коэффициента для пересчета значений освещенности, выраженной в люксах для фотопического зрения, в число фотонов, следует принимать равным $k_{E}=6.1^{-12} \mathrm{~lx} \cdot \mathrm{cm} /$ photon. На рис. 4 кружками отмечены расчетные значения, полученные при таком значении коэффициента пересчета. Эти значения практически совпадают с экспериментальными в области пространственных частот, где находится экстремум ФКЧ. Однако на нижних пространственных частотах расчетные значения превышают данные экспериментов, а на высоких частотах - результаты занижены примерно на 20\%. Как будет показано ниже, одной из причин такого несоответствия расчетных и экспериментальных результатов является использование при расчетах ФКЧ приближенной формулы (26).

Формулы (25) и (26) наглядны и удобны для расчета ОСШ для отдельных гармоник сигнала, а также ФКЧ. Но, как оказалось, эти формулы могут вносить существенные погрешности в результаты. Для оценки погрешности и границ применимости этих приближенных формул были проведены экспериментальные исследования. Для этого производились экспериментальные измерения ФКЧ, значения которой сравнивались с расчетными результатами, полученными по приближенной формуле (26) и более общей формуле, которая имеет вид

$$
S\left(\frac{m}{l_{\varphi x}}, 0\right)=\frac{\left|\tilde{H}_{O S}\left(\frac{m}{l_{\varphi x}}, 0\right)\right|\left|\tilde{H}_{l a t}\left(\frac{m}{l_{\varphi x}}, 0\right)\right|\left|\tilde{H}_{D}\left(\frac{m}{l_{\varphi x}}, 0\right)\right|}{2 \mu^{*} \sqrt{\sigma_{e m n}^{2}+\sigma_{p h m n}^{2}+\sigma_{n m n}^{2}}}
$$

где дисперсии составляющих помех, входящие по знак квадратного корня, определяются по формулам (21), (29) и (30).

Так как погрешность приближения, принятого при выводе формулы (26), зависит от размеров тест-объекта, то исследования выполнялись для двух габаритных размеров, а именно: $0.50 \times 0.29 \mathrm{deg}$ и $1.00 \times 0.57 \mathrm{deg}$. Эксперименты проводились при предъявлении операторам зашумленных изображений, контраст которых варьировался за счет изменения яркости фона в от 88 


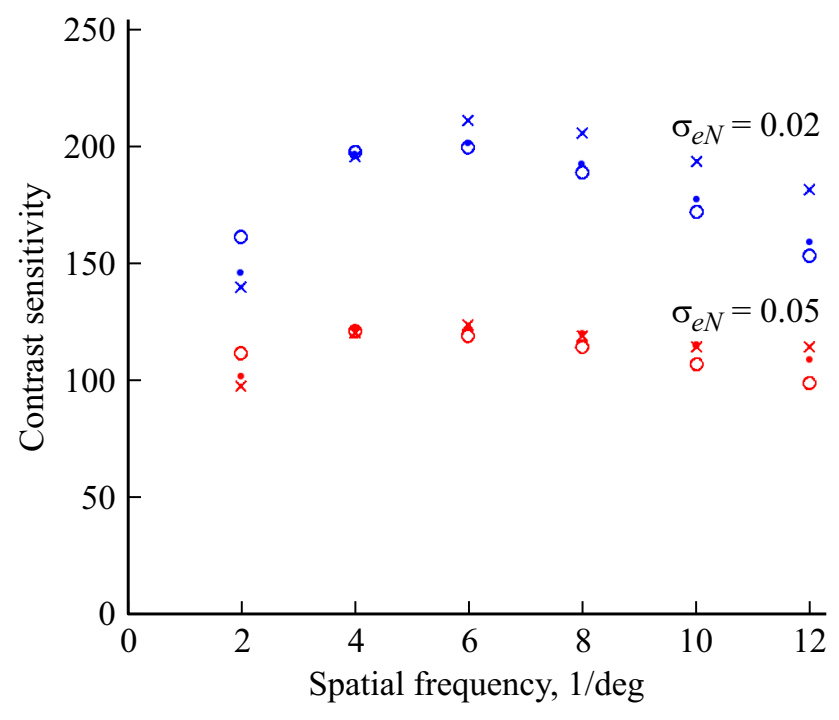

Рис. 5. Расчетные и экспериментальные значения ФКЧ для тест-объекта с размерами $0.50 \times 0.29 \mathrm{deg}: \times-$ экспериментально полученные значения, о - расчетные значения, полученные по приближенной формуле (26), • - расчетные значения, полученные по формуле (33).

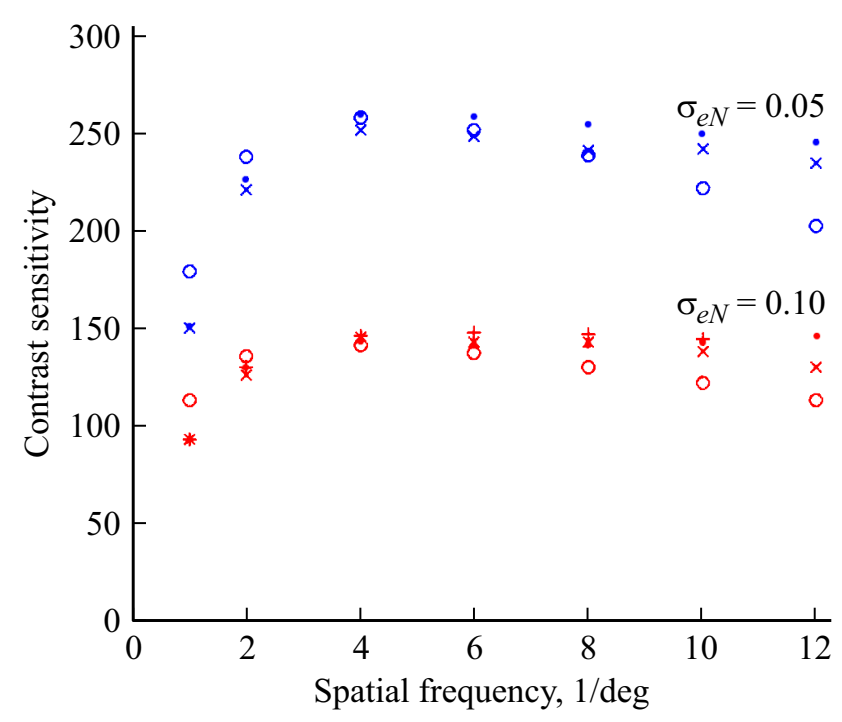

Рис. 6. Расчетные и экспериментальные значения ФКЧ для тест-объекта с размерами $1.00 \times 0.57 \mathrm{deg}: \times-$ экспериментально полученные значения, о- расчетные значения, полученные по приближенной формуле (26), • - расчетные значения, полученные по формуле (33).

до $160 \mathrm{~cd} / \mathrm{m}^{2}$. При расчетах использовались уточненные выше значения параметров математической модели.

На рис. 5 представлены результаты исследований, которые получены для тест-объекта с габаритными размерами $0.50 \times 0.29 \mathrm{deg}$, при добавлении внешней аддитивной помехи со значениями нормированного СКО $\sigma_{e N}=0.02$ и $\sigma_{e N}=0.05$. На рис. 6 представлены результаты исследований, полученные для тест-объекта с габаритными размерами $1.00 \times 0.57 \mathrm{deg}$ при значениях нормированного СКО, равных $\sigma_{e N}=0.05$ и $\sigma_{e N}=0.1$.

На основе анализа результатов, представленных на рис. 5 и 6, можно сделать следующие выводы:

- расчетные значения ФКЧ, полученные по формуле (33), в большей степени соответствуют экспериментальным значениями, чем значения, полученные по приближенной формуле (26);

- погрешность приближенной формулы минимальна в окрестности пространственных частот от 4 до $8 \mathrm{deg}^{-1}$, где находится экстремум ФКЧ.

Кроме этого, следует отметить, что расчетные значения ФКЧ, полученные по формуле (33), заметно отличаются от экспериментальных значений. Причем для тестобъекта с размерами $0.50 \times 0.29 \mathrm{deg}$ расчетные значения отличаются от экспериментов в меньшую сторону, а для тест-объекта с размерами $1.00 \times 0.57 \mathrm{deg}-$ в большую сторону. Для объяснения этого результата, вероятно, следует провести дополнительные исследования.

\section{Заключение}

На основе разработанной методики проведены экспериментальные исследования, которые позволили подтвердить основные теоретические положения многоканальной модели зрительной системы человека и уточнить математические выражения и параметры, используемые в модели. В частности, уточнены значения следующих параметров:

- значения спектральной плотности нейронного шума, которое следует принимать равным $\tilde{R}_{n N}=6.0 \cdot 10^{-9} \mathrm{deg}^{2} \cdot \mathrm{s}$;

- значений параметров, используемых при модельном описании эффекта латерального торможения зрительной системы человека, значения которых приняты равными $r_{\text {lat }}=0.05 \mathrm{deg}$ и $\tau_{1}=2.0 \cdot 10^{-3} \mathrm{~s}$;

- значение коэффициента для пересчета значений освещенности, выраженной в люксах, в число фотонов, которое для фотопического зрения следует принимать равным $k_{E}=6.1^{-12} 1 \mathrm{x} \cdot \mathrm{cm} /$ photon.

Изложенная в материалах работы математическая модель зрительной системы человека-оператора может быть использована для целей проектирования ОЭП наблюдения.

\section{Соблюдение этических стандартов}

Все процедуры, выполненные в исследовании с участием людей, соответствуют Хельсинкской декларации 1964 года и ее последующим изменениям или сопоставимым нормам этики. От каждого из включенных в исследование участников было получено информированное добровольное согласие.

\section{Конфликт интересов}

Авторы заявляют, что у них нет конфликта интересов. 


\section{Список литературы}

[1] Lloyd J.M. Thermal Imaging Systems. NY.: Plenum Press, 1975. (Ллойд Джк. Системы тепловидения. Пер. с англ. Н.В. Васильченко / Под ред. А.И. Горячева. М.: Мир, 1978.)

[2] Malarvizhi D., Lavanya V., Nivethapriya M. Night Vision Technology. [Электронный ресурс] Режим доступа: https://www.researchgate.net/publication/ 320299022_Night_Vision_Technology

[3] Красильников Н.Н. Теория передачи и восприятия изображений. М.: Радио и связь. 1986. 247 с.

[4] Бондарко В.М., Данилова М.В., Красильников Н.Н., Леушина Л.И., Невская А.А., Шелепин Ю.Е. Пространственное зрение. СПб.: Наука, 1999. 218 с.

[5] Krasil'nikov N.N., Krasil'nikova O.I., Shelepin Y.E. // J. Opt. Technol. 2002. V. 69 (5).

[6] Глезер В.Д. Зрение и мышление. Л.: Наука, 1985. 246 с.

[7] Hubel David H. Eye, brain and vision. NY.: Scientific American Library: Distributed by W.H. Freeman, 1988. 240 p.

[8] Barten Peter G.J. Contrast Sensitivity of the Human Eye and Its Effects on Image Quality / Peter G.J. Barten. p. cm. Originally published: Knegsel: HV Press, 1999.

[9] Муравьева С.В., Пронин С.В., Шелепин Ю.Е. // Экспериментальная психология. 2010. Т. 3. № 3. С. 5-20.

[10] Pelli D.G., Raghavan M. Photon and Cortical Noises Limit What We See // Computational and Systems Neuroscience (Cosyne) 2016, February 25-28, 2016 in Salt Lake City. doi 10.7490/f1000research.1115203.1

[11] Gulina Y.S., Koliuchkin V.Ya., Trofimov N.E. // Optical Memory and Neural Networks. 2018. V. 27. N 4. P. 219-234. doi: 10.3103/S1060992X1804001X

[12] Вентиель Е.С., Овчаров Л.А. Теория вероятностей и ее инженерные приложения. М.: Наука, 1988. 480 с.

[13] Rose A. Vision Human and Electronic. NY.: Plenum Press, 1973. $197 \mathrm{p}$.

[14] Луизов А.В. Инерция зрения. М.: Оборонгиз, 1961. 248 с. 